scheidung folgenden Zeit, eben bis längstens zum Inkrafttreten einer Neuregelung, und selbst für diesen Zeitraum hat das Gericht die Anwendungsmöglichkeiten durch das bereits beschriebene zusätzliche Erfordernis einer psychischen Störung und einer besonders hochgradigen Gefährlichkeit beschränkt. Es wäre ohne weiteres möglich gewesen, für die Zeit nach Ablauf dieser Übergangsphase die Anordnung der nachträglichen Unterbringung generell auszuschließen, nicht erst für Taten, die nach Inkrafttreten der Neuregelung begangen werden. Die Begründung für diese Regelung des Referentenentwurfs schreibt dazu lapidar: „Damit wird ... diese rechtlich und tatsächlich problematische Anordnungsform noch so lange fortgeführt, bis der Schutz der Bevölkerung durch den Ausbau insbesondere der vorbehaltenen Sicherungsverwahrung übernommen werden kann“ (ReferentenE S. 16). Es bleibt abzuwarten, ob das Bundesverfassungsgericht damit zufrieden sein wird, ob das der geforderten ,Freiheitsorientierung' (BVerfGE a.a.O. Rn.120) entspricht, zumal die Begründung des Referentenentwurfs selbst formuliert, dass „die Fortgeltung der einschlägigen Regelungen über die vom Urteil des BVerfG erfasste Übergangszeit hinaus“ bestimmt werde.

Insgesamt greift der Referentenentwurf die Vorgaben des Bundesverfassungsgerichts weitgehend angemessen auf und setzt sie in bundesgesetzliche Zielvorgaben um. Manchmal hätte man sich weitergehende und konkretere Regelungen gewünscht, wobei man die Kompetenzverteilung nach der Föderalismusreform berücksichtigen muss, die Bundes- und Landesgesetzgeber zur Entwicklung eines Gesamtkonzepts verpflichtete. Man darf gespannt auf die Umsetzungen in den Ländern in den Gesetzgebungsverfahren, der Praxis der Gerichte und des Justizvollzugs sowie hinsichtlich der Verzahnungen mit den Sozialen Diensten der Justiz und den Freien Trägern sein. Wer sich auf die oben genannten Eckpunkte bezieht, der wird feststellen, dass der Referentenentwurf natürlich Forderungen zur Abschaffung der Sicherungsverwahrung nicht aufnimmt, aber dass er andererseits mehr an Rechtsstaatlichkeit und rationaler Kriminalpolitik beinhaltet, als dies die zurecht gerügte Praxis der Sicherungsverwahrung der letzten Jahrzehnte mit sich brachte. Und schließlich wird man bei aller hier geäußerten Kritik darauf hinweisen können, dass der jetzt vorliegende Referentenentwurf die Forderungen des Bundesverfassungsgerichts bis auf die Übergangsvorschriften ${ }^{1}$ uneingeschränkt berücksichtigt. Das Bundesverfassungsgericht hatte in seinem Urteil vom 4.Mai 2011 die Länder und den Bund verpflichtet, „ein freiheitsorientiertes und therapiegerichtetes Gesamtkonzept für die Unterbringung zu entwickeln “2 und dabei insb. dem Abstandsgebot zum Strafvollzug gerecht zu werden. Das sind große Worte, aber der Entwurf zeigt das Bemühen des Bundesjustizministeriums, die geforderten Elemente einzubringen und deutlich über die seit 1. Januar 2011 geltende Fassung hinauszugehen, die nach dem Urteil des Europäischen Gerichtshofes für Menschenrechte (EGMR) vom 17.12.2009 verabschiedet worden war.

Meine Erwartungen hinsichtlich der Beseitigung der genannten Mängel und Schwächen im Zuge des weiteren Gesetzgebungsverfahrens sind nicht sehr hoch. Es wird nun darauf ankommen, dass an den oben bezeichneten Stellen nicht noch Abschwächungen vorgenommen werden und dass die Länder ihre Teile angemessen umsetzen.

Prof. Dr. Heinz Cornel lehrt an der Alice-Salomon-Hochschule Berlin, ist Präsident des DBH-Fachverbandes für Soziale Arbeit, Strafrecht und Kriminalpolitik und Mitherausgeber der Neuen Kriminalpolitik

\section{Fußnoten}

1 Wie oben beschrieben, widersprechen die Regelungen zwar nicht der Entscheidung des Bundesverfassungsgerichts, sie weiten die Übergangszeit meines Erachtens aber unangemessen aus.

2 vgl. Entscheidung des BVerfG vom 4. Mai 2011(2 BvR 2365/09), Rn 120

\title{
Ein Musterentwurf nicht ohne Wert.
}

\section{Anmerkungen zum ME StVollzG1 vom Autorenkreis des AK StVollzG2}

Johannes Feest

Am 6.9.2011 haben elf Bundesländer einen gemeinsamen Musterentwurf (ME) für ein Landesstrafvollzugsgesetz vorgelegt. Der Entwurf enthält eine ganze Reihe erfreulicher Verbesserungen, leider aber auch einige Rückschritte. Die grundlegenden Schwächen, die schon das alte Gesetz zum Torso gemacht haben (Arbeitsentgelt, Krankenversicherung, Rentenversicherung) behebt er nicht. Es ist mehr als wahrscheinlich, dass die Realität des Vollzuges (zu wenig sinnvolle Angebote, einschließlich produktiver Arbeit) die normativen Fortschritte wieder zunichte machen wird. In einem rauer gewordenen sozialen Klima sind die Chancen zu einem Neuanfang für die meisten Straffälligen ohnehin miserabel.

Die Föderalisierung der Gesetzgebung zum (Erwachsenen-) Strafvollzug in Deutschland schreitet voran. Nachdem Bayern, Nie- dersachsen und Hamburg bereits 2007 gesetzgeberisch tätig geworden waren, haben im Jahre 2009 auch Baden-Württemberg und Hessen eigene Landesstrafvollzugsgesetze erlassen. Jetzt folgen die restlichen Bundesländer (mit Ausnahme Nordrhein-Westfalens), die einen gemeinsamen Musterentwurf eines Strafvollzugsgesetzes (ME) vorgelegt haben. Die Vorlage bietet Anlass zu einer kriminalpolitischen Analyse und einem Vergleich mit dem (Bundes-)Strafvollzugsgesetz (StVollzG) von 1976 sowie den bereits erlassenen Landesgesetzen, die zum Teil erhebliche Rückschritte gegenüber dem früheren Recht aufweisen ${ }^{3}$.

Anders als die bisher im Zuge der Föderalismusreform verabschiedeten Landesgesetze beschränkt sich die Gesetzesinitiative auf die Regelung des Erwachsenenstrafvollzugs ${ }^{4}$. Damit wird nicht nur den 
Gesetzgebungsfristen für den Untersuchungshaft- und Jugendstrafvollzug Rechnung getragen, sondern zugleich die Eigenständigkeit der Regelungsmaterie betont und der Gefahr vorgebeugt, bereits gesetzestechnisch zu einer vereinheitlichenden Vollzugsgestaltung unterschiedlicher Arten von Freiheitszug beizutragen. Insofern geht es ja nicht nur um die formale Beachtung von Abstands- und Trennungsgeboten, sondern um die Aufgabe, gesetzliche Regelungen für den Vollzug der Sicherungsverwahrung, der Jugendstrafe und der Untersuchungshaft zu verabschieden, welche die jeweiligen Besonderheiten ausreichend berücksichtigen. Ein erster positiver Unterschied zwischen dem ME und den Landesgesetzen liegt also bereits darin, dass er nicht der Versuchung unterliegen kann, mit einer umfassenden Regelung zugleich allen Gesetzgebungsaufträgen zu genügen.

Der ME will - an die bewährten Regelungen des StVollzG anknüpfend - das Strafvollzugsrecht nach den Erkenntnissen der Kriminologie und der Rechtsprechung des Bundesverfassungsgerichts weiterentwickeln und neben neuen Schwerpunkten die Vollzugsgestaltung stärker konturieren. Die Themenbereiche sind häufig gekürzt und insgesamt übersichtlicher gestaltet worden. Nicht mehr enthalten sind, neben der besonderen Regelung der Sicherungsverwahrung ( $\mathbb{S}$ 129-135 StVollzG), auch die besonderen Vorschriften zur Unterbringung in einem psychiatrischen Krankenhaus und in einer Entziehungsanstalt (\$S 136-138 StVollzG) sowie zum Datenschutz ( $\mathbb{S}$ 179-187 StVollzG). Während der Maßregelvollzug in einem psychiatrischen Krankenhaus und einer Entziehungsanstalt längst föderal geregelt ist und der Vollzug der Sicherungsverwahrung einer vollständigen Neuregelung bedarf, sollen die besonderen Datenschutzregelungen in die Landesdatenschutzgesetze aufgenommen werden. Für bestimmte Regelungsmaterien wie den gerichtlichen Rechtsschutz besteht keine Zuständigkeit der Länder, sondern weiterhin die konkurrierende Gesetzgebungsbefugnis des Bundes, so dass insofern eine Neuregelung (durch die Länder) von vornherein ausscheidet.

Der ME setzt sich positiv von den Landesstrafvollzugsgesetzen ab. Unterschiede - auch zum StVollzG - finden sich bereits bei der Beschreibung des Vollzugsziels und der Vollzugsaufgabe(n) sowie der zentralen Vollzugsgrundsätze. Während die Landesstrafvollzugsgesetze die Sicherheit der Allgemeinheit zu Lasten des Vollzugsziels der Wiedereingliederung betonen, bleibt der ME der bewährten und verfassungsrechtlich gebotenen Konzeption des StVollzG treu, wonach der Vollzug primär dem Resozialisierungsziel und (nur) sekundär der Vollzugsaufgabe des Schutzes der Allgemeinheit vor weiteren Straftaten dient. Diese Ausrichtung wird in mehrfacher Hinsicht betont: zum einen dadurch, dass der ME anstelle der missverständlichen Überschrift des $\mathbb{2}$ StVollzG („Aufgaben des Vollzugs“) zunächst von einem Ziel („die Gefangenen zu befähigen, künftig in sozialer Verantwortung ein Leben ohne Straftaten zu führen“) und dann erst von einer Aufgabe („,die Allgemeinheit vor weiteren Straftaten zu schützen“) spricht, zum anderen durch wichtige programmatische Ergänzungen der Grundsätze der Vollzugsgestaltung. Nach $\ 3$ Abs. 2 ME wirkt der Vollzug von Beginn an auf die Eingliederung der Gefangenen in das Leben in Freiheit hin. Die Absicht des Entwurfs, entsprechend der Rechtsprechung des Bundesverfassungsgerichts (auch) durch eine frühzeitige und konsequentere Entlassungsvorbereitung Rückfälle zu vermeiden, wird ergänzt durch die Verpflichtung des Vollzugs, den ungünstigen Nebenwirkungen des Freiheitsentzuges wie einer Lebensuntüchtigkeit der Gefangenen entgegenzuwirken. Dazu ist der Angleichungsgrundsatz („Das Le- ben im Vollzug ist den allgemeinen Lebensverhältnissen soweit wie möglich anzugleichen.“) verdeutlicht (,ist“ statt „soll“) und um den Öffnungsgrundsatz in $\mathbb{3}$ Abs. 5 Satz 1 ME ergänzt worden, wonach „der Bezug der Gefangenen zum gesellschaftlichen Leben .. zu wahren und zu fördern“ ist. Die Öffnung des Vollzugs soll in beide Richtungen praktiziert werden: Von „drinnen nach draußen“ beispielsweise durch erweiterte Lockerungsmöglichkeiten (s.u.), aber auch von „draußen nach drinnen“. Ausprägungen dieses Grundsatzes sollen deshalb die (spärliche) Verdoppelung der Besuchszeiten auf mindestens zwei Stunden im Monat ( $\int 26$ Abs. $1 \mathrm{ME}$ ) oder die ausdrückliche Erwähnung des Langzeitbesuchs ( $\mathbb{2 6}$ Abs. $4 \mathrm{ME}$ ) sein. Darüber hinaus müsste der Öffnungsgrundsatz etwa bei der Zulassung neuer elektronischer Kommunikationsmittel wie dem Internet erhebliche Bedeutung bekommen. Dem ausdrücklichen Ziel einer stärkeren Öffnung des Vollzugs dient auch der Grundsatz, dass „Personen und Einrichtungen außerhalb des Vollzugs .. in den Vollzugsalltag einbezogen werden “ sollen ( $\$ 3$ Abs. 5 Satz $2 \mathrm{ME}$ ). Die Stärkung und Ergänzung der Vollzugsgrundsätze ist kriminalpolitisch zu begrüßen. Sie trägt den kriminologischen Erkenntnissen und verfassungsrechtlichen Anforderungen in bemerkenswerter Klarheit Rechnung. Ob sie allerdings auch im Vollzugsalltag und in den Entscheidungen der Strafvollstreckungskammern und -senate zu grundsatzkonformeren Entwicklungen beitragen kann, bleibt abzuwarten. Die bisherigen Erfahrungen mit der Umsetzung der Vollzugsgrundsätze des $\int 3$ StVollzG geben keinen Anlass zu großen Hoffnungen. Von einer erfolgreichen gesetzgeberischen Konzeption wird man aber erst dann sprechen können, wenn den hehren Worten auch Taten folgen.

Erhebliche Abweichungen vom StVollzG, aber auch von den Landesstrafvollzugsgesetzen, finden sich weiter in den Lockerungsvorschriften des ME. Als Lockerungen werden nach der Legaldefinition

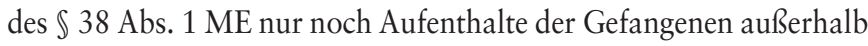
der Anstalt „ohne Aufsicht“ verstanden, so dass eine Ausführung oder Außenbeschäftigung nach $\mathbb{1} 11$ Abs. 1 StVollzG keine Lockerung im Sinne des ME darstellt und deshalb einer gesonderten Regelung $(\mathbb{S} 41 \mathrm{ME})$ bedurfte. Der bisherige Urlaub ( $\mathbb{1} 13$ StVollzG) wird - wie in fast allen Landesgesetzen - wegen des systematischen Zusammenhangs als „Langzeitausgang“ ( $\$ 38$ Abs. 1 Nr. $3 \mathrm{ME}$ ) in die Lockerungsvorschriften einbezogen.

Positiv verändert hat sich in mehrfacher Hinsicht der zeitliche Gestaltungsspielraum der Anstalten bei der Lockerungserteilung: Der mehrtägige Urlaub (sprich Langzeitausgang) ist nicht mehr auf eine bestimmte Anzahl von (Kalender-)Tagen begrenzt. Seine Häufigkeit und Dauer bestimmt sich vielmehr allein nach der Erreichung des Vollzugsziels und den insoweit einschlägigen Gestaltungsgrundsätzen des $\ 3$ Abs. 2, 4 und 5 ME. Die Mindestvollzugszeit für einen Langzeitausgang bei Lebenslänglichen ist von zehn auf fünf Jahre verringert worden $(\$ 38$ Abs. 3 Satz $2 \mathrm{ME}$ ) und ein begleiteter oder unbegleiteter Ausgang ( $\mathbb{3} 38 \mathrm{Abs} .1 \mathrm{Nr} .1$ und $2 \mathrm{ME}$ ) ausdrücklich bis zu 24 Stunden möglich. Im Zuge der Entlassungsvorbereitung vergrößern sich die Gestaltungsspielräume der Anstalten noch einmal. Nach $\ 42$ Abs. 3 ME können Gefangene als „Lockerung sui generis“ in Einrichtungen außerhalb des Vollzugs untergebracht oder ihnen kann ein entlassungsvorbereitender Langzeitausgang von bis zu sechs Monaten gewährt werden.

Die nach dem Willen des ME erweiterten Möglichkeiten der Erprobung in Lockerungen zeigen sich darüber hinaus vor allem in der Veränderung des Prüfungsmaßstabs. An die Stelle der Missbrauchsklausel des $\ 11$ Abs. 2 StVollzG (, wenn nicht zu befürchten ist, dass 
der Gefangene sich dem Vollzug der Freiheitsstrafe entziehen oder die Lockerungen des Vollzuges zu Straftaten missbrauchen werde“) tritt der positiv formulierte Prüfungsmaßstab einer verantwortbaren Erprobung ( $\$ 38$ Abs. $2 \mathrm{ME}$ ). Für einen Zeitraum von sechs Monaten vor der voraussichtlichen Entlassung werden Lockerungserteilungen zusätzlich erleichtert. Danach „sind den Gefangenen die zur Vorbereitung der Eingliederung erforderlichen Lockerungen zu gewähren, sofern nicht mit hoher Wahrscheinlichkeit zu erwarten ist, dass die Gefangenen sich dem Vollzug der Freiheitsstrafe entziehen oder die Lockerungen zu Straftaten missbrauchen werden“ ( $\$ 42$ Abs. 4 ME). Liegen diese - im Vergleich zu $\ 38$ Abs. 2 ME herabgesetzten - Voraussetzungen vor, haben die Gefangenen sogar einen Anspruch auf die zum Zweck der Eingliederung erforderlichen Lockerungen.

Mit diesen Regelungen wird die langjährige Rechtsprechung des Bundesverfassungsgerichts umgesetzt, im Resozialisierungsinteresse der Gefangenen keine unverhältnismäßigen Hürden für Lockerungserteilungen aufzubauen. Darüber hinaus wird zumindest im entlassungsnahen Zeitraum die alte Forderung nach einem Rechtsanspruch der Gefangenen auf Lockerungen verwirklicht. Es bleibt zu hoffen, dass die vorgesehenen Änderungen die seit langem rückläufige Tendenz der Lockerungserteilungen stoppen und zu häufigeren Genehmigungen führen werden. Dass Lockerungen (nur) zur Erreichung des Vollzugsziels gewährt und vor allem in der Entlassungsphase erleichtert werden, dürfte einer unverändert restriktiven Praxis jedenfalls keinen Vorschub leisten. Einem zu engen Verständnis von Vollzugsziel und Eingliederungszweck wird durch die wiederholte Bezugnahme auf die maßgeblichen Vollzugsgrundsätze vorgebeugt.

Eine bedauerliche Änderung betrifft die Aufgabe des offenen Vollzugs als Regelvollzugsform. Anders als noch im StVollzG vorgesehen stellen der offene und der geschlossene Vollzug nunmehr gleichrangige Vollzugsformen dar, weil - so die Begründung - die Unterbringung der Gefangenen allein von deren Eignung abhänge ( $\mathbb{S} 15 \mathrm{ME}$ ). Selbst wenn diese Neuregelung in der Praxis kaum Auswirkungen haben dürfte, da die Umsetzung des früheren Konzepts von den Anstalten und Aufsichtsbehörden konsequent verweigert wurde, bleibt doch die Enttäuschung über den Abschied von einem Kernanliegen der Strafvollzugsreform. Dem Öffnungsgrundsatz hätte es eher entsprochen, auf diese Änderung zu verzichten. Immerhin geht die Neuregelung nicht so weit, den geschlossenen Vollzug wie in Bayern, Hessen und Niedersachsen ausdrücklich zur Regelvollzugsform zu erklären.

$\mathrm{Zu}$ den Vollzugsgrundsätzen gehört auch die Verpflichtung, die unterschiedlichen Bedürfnisse der Gefangenen, insbesondere im Hinblick auf Geschlecht, Alter und Herkunft, bei der Vollzugsgestaltung im Allgemeinen und im Einzelfall zu berücksichtigen $(\mathbb{S} 3$ Abs. $6 \mathrm{ME}$ ). Ob mit dieser allgemein gehaltenen Regelung eine ausreichende Differenzierung etwa für weibliche oder ältere Gefangene gewährleistet ist, bleibt zweifelhaft. Der Verzicht auf besondere Vorschriften für den Frauenstrafvollzug, wie sie (wenn auch in grotesker Verkürzung auf die Mutterrolle) noch in den $\$ \mathbb{S} 76-80$ StVollzG enthalten waren, könnte den unterschiedlichen Interessen und Lebensbedingungen von Männern und Frauen bei der Vollzugsgestaltung und geschlechtsspezifischen Behandlungsansätzen im Sinne eines Gender Mainstreaming widersprechen. Solche Befürchtungen lägen möglicherweise insbesondere dann nahe, wenn die getrennte Unterbringung von männlichen und weiblichen Gefangenen $(\mathbb{S} 10$ $\mathrm{ME})$ nur noch in getrennten Abteilungen, nicht aber besonderen
Frauenanstalten stattfände. Die bisherige Regelung im StVollzG hat die traditionelle Randständigkeit des Frauenvollzugs und sein Schattendasein jedenfalls nicht beseitigt. Es bleibt abzuwarten, ob hierfür allein der neue Hinweis in $\$ 3$ Abs. $6 \mathrm{ME}$ ausreicht.

Der ME greift die auch vom Bundesverfassungsgericht betonte Vorstellung auf, dass die Vollzugsplanung zentraler Bestandteil eines auf Wiedereingliederung gerichteten Vollzuges ist. Der Entwurf sieht insofern eine ganze Reihe von Verbesserungen gegenüber dem StVollzG vor: Grundsätzlich haben alle Inhaftierten einen Anspruch auf Vollzugsplanung. Das ist ein wesentlicher Fortschritt gegenüber dem StVollzG, wonach bei kürzeren Strafen auf eine Vollzugsplanung verzichtet werden kann (und daher meist auch verzichtet wird). Bei Strafen bis zu einem Jahr kann jedoch auf eine umfassende Diagnostik verzichtet und das Augenmerk auf die Umstände gerichtet werden, „deren Kenntnis für eine angemessene Vollzugsgestaltung unerlässlich und für die Eingliederung erforderlich ist“ ( $\$$ 7 Abs. 4 ME). Für die Erstellung eines Vollzugsplans und dessen Fortschreibung sind im ME enge Fristen festgelegt. Warum diese allerdings nur „regelmäßig“ gelten sollen, ist unverständlich, weil damit dem Vollzug die Möglichkeit eröffnet wird, in Einzelfällen weiterhin monate- oder sogar jahrelang ohne Vollzugsplan auszukommen. Die Vorgaben für die Inhalte des Vollzugsplans sind wesentlich erweitert (von acht auf zwanzig Punkte). Besonders erfreulich ist dabei die ausdrückliche Verpflichtung, den voraussichtlichen Entlassungszeitpunkt im Vollzugsplan zu benennen ( $\$ 9$ Abs. 1 Nr. 2 ME) und damit zu einer frühzeitigen Klärung beizutragen. Ausdrücklich aufgeführt sind auch einzelne Therapie-, Trainings- und Ausbildungsmaßnahmen, die (nach $\$ 55 \mathrm{ME}$ ) eine gleichwertige finanzielle Anerkennung wie die Arbeit erhalten sollen.

Ob diese wohlüberlegten Vorgaben eine wirkliche Verbesserung der Vollzugssituation der meisten Gefangenen bringen werden, hängt allerdings entscheidend von der materiellen Ausstattung der jeweiligen Anstalt ab. Wo nichts ist, hat auch der beste Vollzugsplaner sein Recht verloren. Aus den gesetzlichen Vorgaben der Vollzugsplanung einen Anspruch auf bestimmte Ausbildungsbetriebe, Werkstätten, Schulzweige etc. abzuleiten, dürfte jedenfalls schwierig sein.

Der ME will die Notwendigkeit der sozialen Hilfe in allen Phasen des Vollzugs stärker betonen. Dazu werden die Aufgaben sozialer Hilfe nicht mehr wie in den $\mathbb{S}$ 71-75 StVollzG gebündelt dargestellt, sondern in die zeitliche Struktur des Strafvollzugs eingepasst. Nähere Angaben zu den jeweils beteiligten Diensten und zur Qualifizierung der Mitarbeiter des Sozialdienstes sucht man jedoch vergeblich. Die allgemein gehaltene Eingangsvorschrift des $\int 5 \mathrm{ME}$ betont zu Recht auch die Notwendigkeit der Unterstützung bei der Schuldenregulierung, ohne jedoch deutlich einen Anspruch der Gefangenen zu normieren. Zur Hilfe bei der Aufnahme gehört nach $\$ 6$ Abs. $4 \mathrm{ME}$ auch die Beratung zur Abwendung bzw. Verkürzung von Ersatzfreiheitsstrafen und die vom Vollzug trotz obergerichtlicher Entscheidung immer wieder verweigerte Pflicht, auf Nachfrage dem Gefangenen das Strafvollzugsgesetz nebst den zu seiner Ausführung erlassenen Rechts- und Verwaltungsvorschriften zugänglich zu machen. Im Sinne eines erleichterten Übergangs von der Unfreiheit in die Freiheit regeln die $\mathbb{S} \mathbb{S} 44,45 \mathrm{ME}$ deutlicher als das StVollzG die Verlängerung der Entlassungshilfe über den Entlassungszeitpunkt hinaus. Die Möglichkeit, freiwillig in der Anstalt zu verbleiben, wenn bei der Entlassungsvorbereitung Probleme auftreten und die Eingliederung des Gefangenen gefährdet ist, darf jedoch allenfalls als ultima ratio und nicht als Ausweg eines misslungenen Übergangsmanagements verstanden werden. 
Die wesentliche Änderung im Bereich der Unterbringung betrifft die noch häufig praktizierte Mehrfachbelegung der Zellen. Nach $\mathbb{} 11$ Abs. $1 \mathrm{ME}$ werden Gefangene grundsätzlich einzeln untergebracht. Erstaunlicherweise wird die Regelung nicht mit der Wahrung der Menschenwürde oder der Privat- und Intimsphäre der Betroffenen begründet, sondern damit, dass der „elementare Grundsatz“ nicht zuletzt auch dem Schutz der Gefangenen vor Übergriffen diene. Die entscheidenden Unterschiede zur bisherigen Regelung in $\$ 18$ StVollzG liegen weniger im Gesetzeswortlaut als vielmehr im Wegfall der Übergangsvorschrift des $\$ 201$ Nr. 3 StVollzG, der Ausnahmen auch für sog. Alt-Anstalten vorsah.

Der ME will eine Neuausrichtung der Sozialtherapie vornehmen. Anknüpfungspunkt für eine verpflichtende Unterbringung in einer sozialtherapeutischen Einrichtung ist dabei nicht die der Verurteilung zugrunde liegende Straftat, sondern die Verringerung einer erheblichen Gefährlichkeit des Täters durch zu erwartenden Straftaten ( $\int 17 \mathrm{ME}$ ). Weil dies neben Sexual- auch Gewaltstraftaten betrifft, wird die Anzahl der Plätze entsprechend erhöht werden müssen. Da in $\ 93$ Abs. 1 ME nur von „sozialtherapeutischen Abteilungen“ die Rede ist, wird die vom StVollzG gewollte Besonderheit der Sozialtherapie als einer selbständigen Institution im Rahmen des Strafvollzugs aufgegeben. Der Verzicht auf besondere Vorschriften etwa zur Ausstattung und die umstandslose Einordnung der Sozialtherapie in den Katalog aller sonstigen vollzuglichen Maßnahmen lässt befürchten, dass die positive Ausweitung der Verlegungsindikation und die Festlegung der Sozialtherapie als eine auf die Entlassung gerichtete Behandlungsmaßnahme entwertet werden und die sozialtherapeutischen Abteilungen in der Praxis letztlich den Regeln und Strukturen der Gesamtanstalt unterliegen.

Bei der Neuregelung der Arbeit, Ausbildung und Weiterbildung werden schulische und berufliche Qualifizierungsmaßnahmen $(\mathbb{S} 21$ $\mathrm{ME}$ ) vor der Arbeit ( $\$ 22 \mathrm{ME}$ ) genannt. Dadurch wird ihre resozialisierende Bedeutung neben arbeitstherapeutischen Maßnahmen

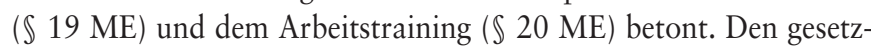
geberischen Anspruch einer besseren Verzahnung von Vollzug und „Außenwelt“ konkretisiert $\$ 21$ Abs. $4 \mathrm{ME}$, wonach die Anstalt in Zusammenarbeit mit außervollzuglichen Einrichtungen dafür Sorge zu tragen hat, dass begonnene Qualifizierungsmaßnahmen nach der Haft fortgesetzt werden können, wenn ein Abschluss während der Haftzeit nicht möglich war. Mit der Streichung der traditionellen Arbeitspflicht wird die Selbstbestimmung der Gefangenen gestärkt und die häufig gesetzeswidrige Zuweisung von Arbeitsplätzen primär an „pflegeleichte“ statt an besonders resozialisierungsbedürftige Gefangene beendet. Dennoch kann - anders als in der Begründung des ME - von einer „freiwilligen“ Arbeit angesichts der Rahmenbedingungen der Haft nicht die Rede sein.

Bei den Vergütungsregelungen sind demgegenüber keinerlei Fortschritte erkennbar. Die Angemessenheit der Entlohnung von Gefangenenarbeit ist sogar noch problematischer geworden, weil die monetäre Vergütung unverändert bleibt ( $\$ 55 \mathrm{Abs}$. $2 \mathrm{ME}$ ) und der nicht-monetäre Teil ( $\$ 43$ Abs. 6 StVollzG) ersatzlos wegfallen soll. Hinzu kommt die Ungleichbehandlung mit den Inhaftierten anderer Bundesländer, deren Regelungen sich nicht unerheblich unterscheiden. Allerdings tragen jedoch weitere Änderungen wie die bereits erwähnte finanzielle Anerkennung (etwa) für die Teilnahme an einzeloder gruppentherapeutischen Maßnahmen ( $\mathbb{5} 5$ Abs. $1 \mathrm{ME}$ ), die Abschaffung des Überbrückungsgeldes oder die ausdrückliche Regelung zweckgebundener Einzahlungen $(\$ 60 \mathrm{ME}$ ) zu einem insgesamt positiven Fazit bei. Dass die Gefangenen auch nach dem ME nicht in die Kranken- und Rentenversicherung einbezogen werden, bleibt ein sozialpolitischer Skandal, war aber nicht anders zu erwarten.

Gespannt konnte man darauf sein, wie der ME mit dem klassischen Repressionsinstrumentarium der Sicherungs- und der Disziplinarmaßnahmen umgehen würde. Das Ergebnis ist durchwachsen:

Die rechtsstaatliche Forderung nach einem Katalog von Disziplinartatbeständen wird zwar erfüllt, aber durch eine Generalklausel wieder aufgeweicht und entwertet. Zwei besonders resozialisierungsfeindliche Strafmaßnahmen sollen wegfallen: Der Entzug des Lesestoffs und die Beschränkung des Verkehrs mit Personen außerhalb der Anstalt. Dafür soll aber der „Entzug des Aufenthalts in der Gemeinschaft" als Disziplinarmaßnahme von vier Wochen auf drei Monate ausgedehnt werden ( $\$ 86$ Abs. 2 Ziff. $4 \mathrm{ME}$ ).

Leider hat der ME sich nicht zur Abschaffung des „Knasts im Knast“, nämlich der Disziplinarmaßnahme des Arrests, entschließen können. Die Begrenzung dieser völligen Absonderung von anderen Gefangenen auf vier Wochen konnte bisher durch unbegrenzte Sicherungsmaßnahmen (Absonderung, Einzelhaft) unterlaufen werden. Hier enthält der ME begrüßenswerte Einschränkungen und Kontrollmöglichkeiten. So sind künftig alle Formen der Absonderung, einschließlich der Unterbringung im besonders gesicherten Haftraum, unverzüglich der Aufsichtsbehörde zu melden, wenn sie mehr als drei Tage andauern. Eine Absonderung von mehr als 30 Tagen im Jahr bedarf sogar der Zustimmung der Aufsichtsbehörde. Gleiches soll für die Fesselung gelten. Damit geht der ME über alle bestehenden Landesgesetze hinaus und trägt langjährigen Forderungen der Literatur Rechnung.

Negativ fällt auf, dass auch im ME einzelnen Gefangenen der tägliche Aufenthalt im Freien („Freistunde“, „Hofgang“) im Namen der Sicherheit entzogen werden darf. Das entspricht zwar der Regelung des Strafvollzugsgesetzes und der fünf vorhandenen Ländergesetze, es widerspricht aber Nr. 27.1 der Europäischen Gefängnisregeln, wonach (ohne Einschränkung) „jeder Gefangene“ jeden Tag Gelegenheit haben muss, sich mindestens eine Stunde im Freien zu bewegen. Das CPT hat in seinem Bericht über den Besuch in Deutschland im Dezember 2005 erneut die Empfehlung ausgesprochen, das Verbot des täglichen Aufenthalts im Freien als besondere Sicherungsmaßnahme aufzuheben (CPT Bericht 2006, Rn. 89). Warum dies nicht geschieht, wird im ME nicht erläutert.

Neu hinzugekommen ist schließlich eine Bestimmung über „Maßnahmen zur Feststellung von Suchtmittelgebrauch“ (\$ $76 \mathrm{ME})$. Eine solche Vorschrift findet sich in allen bereits vorhandenen Landesgesetzen. Danach besteht über die Notwendigkeit einer Rechtsgrundlage für Urinkontrollen u.ä. offenbar Konsens. Mehr als problematisch ist allerdings die gesetzliche Vermutung, wonach von der Verweigerung der Mitwirkung an solchen Maßnahmen auf den Gebrauch illegaler Drogen geschlossen werden darf.

Der Erfolg der Gesetzesinitiative wird nicht zuletzt davon abhängen, ob die betroffenen Länder bereit sind, den Strafvollzug endlich mit den notwendigen sachlichen und personellen Mitteln auszustatten. Der ME weist deshalb zu Recht darauf hin, dass zur Umsetzung des Gesetzes finanzielle Mehraufwendungen unumgänglich sind. Dies betrifft insbesondere die angekündigte Verbesserung des Behandlungsangebots, die Ausweitung der Sozialtherapie, die vorgesehenen Qualifizierungsmaßnahmen und die finanziellen Anreize für die Teilnahme an Vollzugsmaßnahmen, die zur Erreichung des Vollzugsziels zwingend erforderlich sind. Bislang scheiterten Reformbemühungen 
immer wieder auch daran, dass der Vollzug zwar mit hohen Erwartungen konfrontiert, ihm aber nicht die zu ihrer Erfüllung erforderlichen Mittel zur Verfügung gestellt wurden. Die kleinlichen Sparbemühungen, die neben den anspruchsvollen Zielsetzungen den Entwurf durchziehen, verheißen insofern nichts Gutes. So ist in \62 Abs. 2 ME die bislang im StVollzG fehlende Rechtsgrundlage für eine generelle Kostenbeteiligung der Gefangenen an den Aufwendungen der Gesundheitsfürsorge (entsprechend der Beteiligung gesetzlich Versicherter) normiert worden. Damit wird das Ziel der Kostendämpfung im öffentlichen Gesundheitswesen ungeachtet aller tatsächlichen Unterschiede auf den Strafvollzug übertragen und der Grundsatz freier Heilfürsorge trotz fehlender finanzieller Mittel der Gefangenen aufgegeben. Weitere Kostenregelungen finden sich in $\ 63$ Abs. $3 \mathrm{ME}$, wonach Gefangene an den Betriebskosten der in ihrem Gewahrsam befindlichen Geräte beteiligt werden können und in $\ 41$ Abs. 1 Satz 3 ME, wonach den Gefangenen die Kosten einer in ihrem Interesse erfolgten Ausführung auferlegt werden können, soweit dies die Behandlung oder Eingliederung nicht behindert. Der angespannten Haushaltslage dürfte auch die Einschränkung der gemeinsamen Unterbringung von Müttern mit Kindern nur bis zur Vollendung von deren zweitem Lebensjahr geschuldet sein. Wer aber meint, in diesen Bereichen Kleinstbeträge einsparen zu können, wird kaum den politischen Willen und die Kraft zu größeren Investitionen in den Strafvollzug aufbringen, wenn auch noch erhebliche Ausgaben für die reformierte Sicherungsverwahrung drohen.

Der ME unterscheidet sich insgesamt wohltuend von den bisher erlassenen Landesstrafvollzugsgesetzen. Wenn er in dieser Form von den Ländern auch konsequent umgesetzt und nicht noch durch
Änderungen und restriktive Verwaltungsvorschriften verwässert wird, wären auch die im Zuge der Föderalismusreform geäußerten Befürchtungen, ein Landesstrafvollzug führe zum „Flickenteppich“ und zu einem „Wettbewerb der Schäbigkeit“ zumindest teilweise gebannt. Von den großen Hoffnungen der Strafvollzugsreform ist der ME allerdings meilenweit entfernt ${ }^{5}$. Offensichtlich sind die Zeiten aber so, dass man sich schon freut, wenn es nicht ganz so schlimm kommt wie befürchtet.

Der Autor ist em. Professor in Bremen

\section{Fußnoten}

1 Der Text des Musterentwurfs findet sich auf den Webseiten der beteiligten Ministerien (Nachweise auf www.strafvollzugsarchiv.de).

2 Redaktion: Johannes Feest und Wolfgang Lesting (vgl. die Beiträge einzelner AutorInnen des AK StVollzG auf der Webseite des Strafvollzugsarchivs www.strafvollzugsarchiv.de).

3 Vgl. Feest/Lesting (Hrsg.) AK-StVollzG, 6. Aufl.; Köhne NStZ 2009, 130.

4 Alle bisherigen Landesgesetze hatten auch die Sicherungsverwahrung einbezogen, Baden-Württemberg, Bayern, Hamburg und Niedersachsen darüber hinaus den Jugendstrafvollzug. Zwei Bundesländer (Baden-Württemberg und Niedersachsen) hatten sich an einem auch die Untersuchungshaft umfassenden allgemeinen Justizvollzugsgesetz versucht. Allerdings hat Hamburg bereits im Jahre 2009 die Problematik einer gemeinsamen gesetzlichen Behandlung von Jugendlichen und Erwachsenen eingesehen und die Gesetze wieder getrennt. Durch die Entscheidung des Bundesverfassungsgerichts vom 4.5.2011 müssen jetzt jedoch auch eigene Gesetze für die Sicherungsverwahrung geschaffen werden, sodass alle vorausgeeilten Bundesländer nachsitzen müssen.

5 Feest, Das Strafvollzugsgesetz von 1976: eine überholte Reform? KrimZ (Hrsg.) Justizvollzug und Strafrechtsreform im Bundesstaat. Wiesbaden 2011.

\section{Was versteht man unter „Polizeiwissenschaft"- Eine programmatische Standortbestimmung}

Joachim Kersten

Im Jahr 2005 wurde das Gesetz über die Deutsche Hochschule der Polizei (DHPOL) und ihren Masterstudiengang von Bund und Ländern verabschiedet. Demnach hat diese Institution „die Polizeiwissenschaft durch Forschung, Lehre, Studium und Weiterbildung zu pflegen und zu entwickeln“ und insofern macht man sich über die Grundlage und die Perspektive der Disziplin Gedanken. Auch an regulären Universitäten gibt es Lehrstühle, die diese Bezeichnung führen (Uni Bochum, Uni Witten-Herdecke), jedoch in weitaus weniger anspruchsvollem Rahmen.

Polizeiwissenschaft wird wohl zunächst einmal, will sie dem Wortteil „-wissen-schaft“ gerecht werden, systematisch rational überprüfbare Ziele verfolgen und empirisch be- legen müssen. Zudem müssten in Forschung und Lehre Tätige ein entsprechendes Profil (akad. Grade, Veröffentlichungen) aufweisen. Dem Wortteil „Polizei“ zufolge erwartet man von diesem Wissenschaftszweig, dass eine wissenschaftlich informierte und trainierte Polizei, insbesondere in den Führungsetagen, zur Aufgabenstellung gehört. Weiterhin muss eine moderne demokratische Polizei als Wissensorganisation (knowledge organization) aufgefasst werden. Wissen über die Gesellschaft und die Rolle der Organisation sowie der Berufskultur macht nicht handlungsunfähig wie es gelegentlich von den erklärten Wissenschaftsabstinenzlern in der Polizeiführung behauptet wird. Polizeiliches Handeln wird durch Wissenschaft professioneller (,Know- ledge kills action vs. Knowledge skills action ") und somit wird überhaupt langfristig die Handlungsfähigkeit der Polizei gesichert (Tops 2009).

Die traditionelle Didaktik berufsschulmäBiger Polizeilehre gehorcht dem Prinzip „Polizisten lernen von Polizisten, was Polizisten von Polizisten gelernt haben“. Das ist sicherlich nicht mehr zeitgemäß. Man wird sich aber in den „Praxisfächern“ weiterhin auf Kompromisse einlassen müssen, solange noch Führungsakademiesolventen den Fachgebieten vorstehen, die nicht von Universitätsprofessoren geleitet werden. Lehrende mit Erfahrung in der Wissenschaft (Masterarbeiten, zukünftig auch Doktorarbeiten) werden erst in einigen Jahren diese Stellen übernehmen können. 